\section{Brain, Behavior and Evolution}

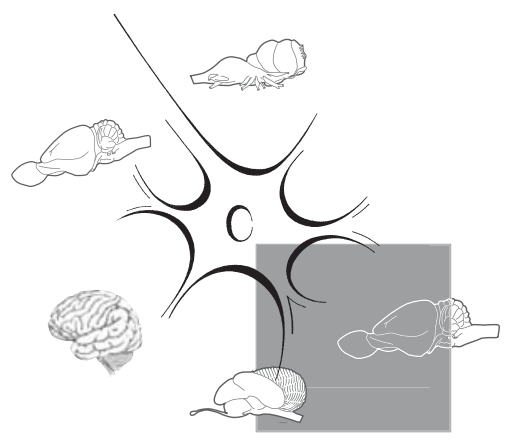

Despite a century of investigation [Smith, 1919; Holmgren, 1922; Johnston, 1923; Northcutt and Kaas, 1995; Karten, 1997; Butler and Hodos, 2005; Striedter, 2005], the evolutionary origins of the cerebral cortex remain intensely disputed [Karten, 1997; Wang et al., 2010; DugasFord et al., 2012; Suzuki et al., 2012]. As brain tissue does not fossilize, comparative neurobiologists infer ancestral forms from living animals. Unfortunately, one of the earliest branches of the mammalian lineage, Eutriconodonta, became extinct at the end of the Cretaceous period (approx. 145-66 Mya). Thus, to gain insights into the evolutionary origin of the isocortex, which already existed in our most recent common ancestor with monotremes, we need to compare living sauropsids (birds and reptiles) with mammals, two clades separated by more than 300 million years of independent evolution in diverse ecological niches. Numerous studies have noted similarities in connectivity and gene expression between the layers of the mammalian isocortex and the nuclear structures of the avian pallium [Karten, 1997; DugasFord et al., 2012]. However, these similarities are seemingly inconsistent with homologies inferred from morphogenetic developmental data. Furthermore, gene ex-
Published online: September 27, 2013

\title{
Things Change: How Comparative Transcriptomics Suggest the Pallium Has Evolved at Multiple Levels of Organization
}

\author{
T. Grant Belgard ${ }^{a} \quad$ Juan F. Montiel ${ }^{b}$ \\ a Semel Institute for Neuroscience and Human Behavior, University of California, \\ Los Angeles, Calif., USA; ${ }^{b}$ Center for Biomedical Research, Facultad de Medicina, \\ Universidad Diego Portales, Santiago, Chile
}

pression has been used to support a wide variety of mutually contradictory homologies between isocortical laminae and various sectors of the avian pallium. It is in this context that we aimed to conduct an unbiased gene expression study using an objective analytical method [Belgard et al., 2013]. Because adult brain regions differ in their transcriptomes, we dissected several structures of both controversial and noncontroversial homology in adult chickens and mice, and then sequenced and compared their transcriptomes. Although we would have liked to look at examples throughout brain development, young embryonic brain regions contain so little RNA that this approach was not feasible with the technology available. So instead, we sequenced RNA from pooled samples representing several adult structures.

The results of our study surprised us, and caused us to conclude that homologous developmental fields can yield staggeringly different adult forms, and that highly similar adult characteristics can arise from nonhomologous developmental fields. Moreover, homologous genes may underlie some of the physiologically comparable functions of these analogous adult regions. Although homologous sets of genes are usually expressed in cells that develop from homolo- gous morphogenetic fields, this may not be the case in the pallium. We believe that there may be different sets of plausible pallial homologies at two (or more) distinct levels of cellular organization: the phylogenetically continuous developmental lineage and the key underlying genetic programs that distinguish one cell type from another. We argue that the latter level of homology is plausible, but cannot be established until the underlying cause of this similar expression is understood.

\section{Comparative Transcriptomics}

Our dissections isolated the dorsal and lateral cortical laminae [Belgard et al., 2011], hippocampus, striatum, pallial amygdala and claustrum/endopiriform complex from mice, and the hippocampus, hyperpallium, mesopallium, nidopallium, arcopallium, dorsolateral corticoid area and striatum from chickens. For each species, these structures were dissected from serial sections along the anteroposterior axis, and dissections from all members of each species were then pooled into one sample per species per region for sequencing. We extracted and deeply sequenced polyadenylated transcriptomes from each

\section{KARGER}

E-Mail karger@karger.com

www.karger.com/bbe (c) 2013 S. Karger AG, Base

0006-8977/13/0823-0150\$38.00/0
T. Grant Belgard

Semel Institute for Neuroscience and Human Behavior, University of California 2309 Gonda Building, 695 Charles E Young Drive South Los Angeles, CA 90095-1761 (USA)

E-Mail grant@grantbelgard.com 
region, and then compared the expression patterns of the most highly expressed genes with established one-to-one orthologs between the two species.

After confirming our dissections and gene expression quantifications with in situ hybridizations of the most specific genes, we conducted four in silico analyses to search for transcriptomic similarities above those expected by chance. We repeatedly found significant and strong similarities between the striatum samples. Likewise, we repeatedly found significant, though weaker, similarities between the hippocampus samples. This was unsurprising, as these structures are of uncontroversial homology between birds, and mammals and retain some readily apparent anatomical and functional similarities. Things were more complicated when we turned to other structures. In most cases, there were no detectable similarities at all.

One exception was a weak but still highly statistically significant overlap of genes expressed in chicken nidopallium and mouse layer IV, areas that receive thalamic input in both species. This expression overlap included some of the genes independently found by Dugas-Ford et al. [2012] to have similar expression patterns in birds and mammals, but our interpretation of this similarity is more nuanced. First, the overlap includes just a handful of genes, not dozens. Second, upon further examination with our own in situ hybridizations and using publicly available data, we found that these genes tend also to be expressed elsewhere in the brain. Third, the genes are generally not expressed together outside of the pallium. Although the expression of some genes may be induced in response to thalamic input, or by generally receiving rapid-fire sensory information, we do not believe this to be the case for the set of genes as a whole, because there was so little overlap in the expression of these genes outside of the structures in question. In conclusion, we suggest that the similarity in expression of some of these genes might be the result of convergent evolution. Alternatively, the similarity may derive from an ancestral pallial gene expression program that existed in the stem amniote, but was expressed in cells on a different developmental lineage from where it is now expressed in extant birds or mammals. This may be plausible considering that the timing and location of some gene expression programs can be experimentally modified by a single genetic change [Carroll, 2008].

\section{Limitations}

To avoid overinterpretation, the limitations of this study must be considered [Belgard et al., 2013]. First, available resources limited the number of dissected structures. Still, we did strive to collect the regions that best represented the most commonly proposed homologies. Second, these transcriptomes represent a weighted average of all cells in a region and could be affected by cellular heterogeneity. For this reason, we implemented analyses that should be less susceptible to such heterogeneity. Third, samples were pooled from many mice and chickens; thus, we rely on pooling to smoothen interindividual variability. Although this may be insufficient for highly variable genes, good marker genes should be consistent between individuals. We also validated the expression of these top genes by in situ hybridization. Fourth, the quality of the RNA in a few samples was poor. For this reason, we redid the analysis without those samples, and found consistent results; however, sample quality would be expected to affect some analyses. Fifth, we were concerned that the specific way that we divided the regions (for example, the individual layers of cortex) could affect the analysis. Thus, we redid our analyses combining different subsets of regions in ways that reflected various proposed homologies. We still found nothing. Sixth, although simulations and the nature of our positive results suggest that our analyses are sufficiently powered to identify even modest similarities, there may be weaker similarities among homologous regions that our analyses could not detect.

\section{Some Insights from the Transcriptomic Organization of the Telencephalon}

The key finding of our study is not that similarities between avian and mammalian pallium do not exist outside of the hippocampus, but that they are relatively weak. That is why we suggest that supposed marker genes in adults cannot be used with confidence to support one claim of homology over another. In particular, we note that Dugas-Ford et al. [2012] and Suzuki et al. [2012] compared expression patterns for a relatively small number of genes and drew conclusions that differ from one another. Indeed, our data suggest there are genes that could be used to support many different patterns of interest.
Based on our analyses, we speculate that, in the highly diverged portions of the pallium, the adult patterning of molecules might better reflect function than homology. In some respects, this is unsurprising. The function of the brain is ultimately imparted by its connectivity and physiological properties, even if these are guided by development. In particular, we know that pallial connectivity exhibits differences between mammals and birds. Whereas somatosensory thalamocortical projections in mammals target mainly the dorsal pallium-derived isocortex, birds have more widely distributed projections extending to both dorsal and ventral pallial sectors [Aboitiz et al., 2002; Csillag and Montagnese, 2005]. We also know that great hodological differences can be driven by single molecular changes [Simpson et al., 2009]. Most hodological changes would likely be deleterious. Still, the fact that some people function relatively normally with considerable differences in their neuroanatomy and connectivity (consider the minor phenotype of some individuals having complete agenesis of the corpus callosum [Chiappedi et al., 2012]) suggests that neural plasticity is sometimes sufficient to largely compensate for such changes. A variant that causes an anatomically and hodologically large but phenotypically relatively neutral change might drift to fixation in a population. With a change in connectivity, the evolutionary constraints on adult gene expression would likely change, and a drift-dominated transcriptomic paradigm with persistent negative selection on the expression of key genes as well as spurts of positive selection could act over hundreds of millions of years, resulting in significant divergence of gene expression patterns. For this reason, we suggest that the genes associated with pallial analogies (i.e. regions sharing similar hodology and physiology but derived from nonhomologous developmental fields) might be as important an object of study as homology in the investigation of cortical evolution.

If one could identify the genetic differences between the two clades that are responsible for this apparent convergence in expression in functionally analogous but developmentally distinct areas, one could potentially determine experimentally if these mutations are sufficient to redirect thalamic efferents to the expressing cells in a species from the other clade. Such studies would provide a more complete under- 
standing of pallial evolution, leveraging the modern evolutionary synthesis and evolutionary developmental biology.

\section{Conclusion}

Both of the dominant ideas on homology - cell-type homology and field homology - appear insufficient by themselves. Field homology [Puelles and Medina, 2002] focuses on the idea that adult structures can be tracked to specific developmental stages, where similarities arise from a conserved embryonic design (Bauplan). Although the adult gene expression data do not contradict this idea, it appears that adult molecular profiles on the whole do not reflect these developmental field homologies in the most divergent pallial sectors. Although we maintain that developmental comparisons are fundamental to understanding the ontogenetic dynamics underlying the origin of adult structures [Medina et al., 2013], one must acknowledge the astonishing diversification of adult amniote forms. Cell-type homology, in contrast, emphasizes similarity in connectivity regardless of developmental origin [Karten, 1969; 2013], as well as some similarities in gene expression [DugasFord et al., 2012]. Our molecular data do reveal some similarity between the pallial regions in birds and mammals, but this similarity is weak in the context of a global transcriptome. Our data are most fully consistent with the proposal by Striedter and Northcutt [1991] that homology can exist at multiple levels of biological organization, as homologous molecular mechanisms could generate characters from nonhomologous developmental fields that have similar properties.

In conclusion, we suggest that concentrating on homologous key functional genes and changes in their cis- and transregulatory factors will help us understand the dramatic divergence of mammalian and avian pallia. For example, one of the most highly interconnected genes from our study has been proposed to participate in thalamocortical innervation [Jabaudon et al., 2012]. If such studies were extended across species, they would supplement, rather than supplant, homologies of developmental fields inferred by morphogenetic data. We suggest that homologies should best be drawn with the most precise description possible. For example, an analogous character could be developed using homologous genes with converging expression patterns. We would say this character is a homoplastic result of convergence [Northcutt, 1984]. In contrast, if an analogous character were developed using a unitary homologous genetic program, the expression of which moved from one place to another, we would recognize this as a deep homology. Until the regulatory relationships of these genes are better understood, we reserve judgment on the possible deep homology of pallial thalamic recipients.

\section{Acknowledgements}

This work was supported by a Marshall Scholarship and NIH-Oxford-Cambridge Scholarship to T.G.B. and a Becas Chile Postdoctoral Fellowship to J.F.M.

\section{References}

-Aboitiz F, Montiel J, Morales D, Concha M (2002): Evolutionary divergence of the reptilian and the mammalian brains: considerations on connectivity and development. Brain Res Rev 39:141-153.

- Belgard TG, Marques AC, Oliver PL, Abaan HO, Sirey TM, Hoerder-Suabedissen A, GarcíaMoreno F, Molnár Z, Margulies EH, Ponting CP (2011): A transcriptomic atlas of mouse neocortical layers. Neuron 71:605-616.

- Belgard TG, Montiel JF, Wang WZ, García-Moreno F, Margulies EH, Ponting CP, Molnár Z (2013): Adult pallium transcriptomes surprise in not reflecting predicted homologies across diverse chicken and mouse pallial sectors. Proc Natl Acad Sci USA 110:13150-13155.

Butler AB, Hodos W (2005): Comparative Vertebrate Neuroanatomy, ed 2. Hoboken, WileyLiss.

-Carroll SB (2008): Evo-devo and an expanding evolutionary synthesis: a genetic theory of morphological evolution. Cell 134:25-36.

-Chiappedi M, Fresca A, Baschenis IM (2012): Complete corpus callosum agenesis: can it be mild? Case Rep Pediatr 2012:752751.

Dugas-Ford J, Rowell JJ, Ragsdale CW (2012): Cell-type homologies and the origins of the neocortex. Proc Natl Acad Sci USA 109: 16974-16979.

Holmgren N (1922): Points of view concerning forebrain morphology in lower vertebrates. J Comp Neurol 34:391-459.

Jabaudon D, Shnider SJ, Tischfield DJ, Galazo MJ, Macklis JD (2012): ROR $\beta$ induces barrel-like neuronal clusters in the developing neocortex. Cereb Cortex 22:996-1006.

Johnston JB (1923): Further contributions to the study of the evolution of the forebrain. J Comp Neurol 35:337-481.

Karten HJ (1969): The organization of the avian telencephalon and some speculations on the phylogeny of the amniote telencephalon. Ann NY Acad Sci 167:164-179.

Karten HJ (1997): Evolutionary developmental biology meets the brain: the origins of mammalian cortex. Proc Natl Acad Sci USA 94: 2800-2804.

Karten HJ (2013): Neocortical evolution: neuronal circuits arise independently of lamination. Curr Biol 23:R12-R15.

Csillag A, Montagnese CM (2005): Thalamotel- Medina L, Abellán A, Desfilis E (2013): A neverencephalic organization in birds. Brain Res Bull 66:303-310. ending search for the evolutionary origin of the neocortex: rethinking the homology concept. Brain Behav Evol 81:150-153.

Northcutt RG (1984): Evolution of the vertebrate central nervous system: patterns and processes. Am Zool 24:701-716.
Northcutt RG, Kaas JH (1995): The emergence and evolution of mammalian neocortex. Trends Neurosci 18:373-379.

Puelles L, Medina L (2002): Field homology as a way to reconcile genetic and developmental variability with adult homology. Brain Res Bull 57:243-255.

- Simpson TI, Pratt T, Mason JO, Price DJ (2009): Normal ventral telencephalic expression of Pax6 is required for normal development of thalamocortical axons in embryonic mice. Neural Dev 4:19.

Smith GE (1919): A Preliminary Note on the Morphology of the Corpus Striatum and the Origin of the Neopallium. J Anat 53:271-291.

Striedter GF (2005): Principles of Brain Evolution. Sunderland, Sinauer Assoc. Inc.

-Striedter GF, Northcutt RG (1991): Biological hierarchies and the concept of homology. Brain Behav Evol 38:177-189.

-Suzuki IK, Kawasaki T, Gojobori T, Hirata T (2012): The temporal sequence of the mammalian neocortical neurogenetic program drives mediolateral pattern in the chick pallium. Dev Cell 22:863-870.

-Wang Y, Brzozowska-Prechtl A, Karten HJ (2010): Laminar and columnar auditory cortex in avian brain. Proc Natl Acad Sci USA 107:12676-12681. 